\title{
La ciencia y el mundo físico de acuerdo a W.T. Stace
}

\section{Science and the physical world according to W.T. Stace}

\author{
Vicente Aboites \\ Centro de Investigaciones en Óptica, México \\ Gilberto Aboites \\ CISE, Universidad Autónoma de Coahuila, México
}

Resumen: Se presenta y expone el argumento de W. T. Stace sobre el realismo, señalando no que éste sea falso sino solamente que no hay absolutamente ninguna razón para considerar que sea verdadero y por tanto no tenemos por qué creerlo. En seguida esto se aplica a la discusión de la pregunta: ¿Cómo sabemos que los átomos existen? Para lo cual se hace referencia a algunas de las respuestas científicas más importantes conocidas que son en orden cronológico: 1) La ley de las proporciones definidas o Ley de Proust, 2) la teoría cinética de los gases, 3) el movimiento Browniano y, 4) imágenes de microscopio de efecto túnel. Concluyendo que, por remoto que parezca, existe la posibilidad lógica de que las no concebidas propuestas teóricas expliquen los resultados observacionales actuales sin requerir de la hipótesis atómica.

Palabras clave: W.T. Stace, filosofía de la ciencia, teoría atómica.

Abstract: Here It's presented and explained the argument of W.T. Stace about realism, pointing out not that this is false but only that there is absolutely no reason to consider it to be truth and therefore we don't have to believe it. Immediately this is applied to the discussion of the question: How do we know atoms exist? In order to do this, reference is made to some of the most important known scientific answers which 
in chronological order are: 1) Proust Law of definite proportions, 2) kinetic gas theory, 3) Brownian motion, and, 4) Tunnel effect microscope images. It is concluded that, as remote as it may seem, there is a logical possibility that not yet imagined theoretical proposals may be able to explain the current observational results without requiring the atomic hypothesis.

Keywords: W.T. Stace, Philosophy of science, Atomic theory.

\section{Introducción}

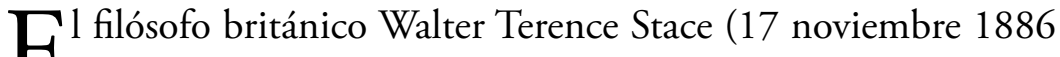
C-2 Agosto 1967) trabajó como profesor de filosofía en la Universidad de Princeton de 1932 hasta su retiro en 1955, pero siguió asociado a dicha universidad como Profesor Emérito. Sus primeros libros fueron escritos cuando trabajo en Ceylán (actualmente Sri Lanka), A Critical History of Greek Philosophy (1920), The Philosophy of Hegel: A Systematic Exposition (1924) y The Meaning of Beauty (1929). Posteriormente siguió la tradición empirista de David Hume, George Edward Moore, Bertrand Russell y Henry Habberley Price. Sin embargo, como en seguida se muestra, a diferencia de otros filósofos, para Stace el empirismo no estaba limitado a proposiciones que es posible demostrar. Adicionalmente Stace es considerado un pionero en el estudio filosófico del misticismo, de hecho muchos consideran su obra Mysticism and Philosophy (1960) como su libro más importante. El trabajo desarrollado por Stace en Princeton entre las décadas de 1930 y 1950 muestra una fuerte influencia de fenomenalismo, que es una forma radical del empirismo (no deberá confundirse con fenomenología). En el primer libro que publicó estando en Princeton, The Theory of Knowledge and Existence (1932), propone una epistemología empírica en donde intenta, "trazar los pasos lógicos a través de los cuales la mente iniciando con lo que le es dado, concluye y justifica su creencia en un mundo externo". Este libro puede ser visto como 
una crítica al pragmatismo. Su trabajo refutando al realismo puede verse de modo paralelo a otra famosa refutación, la del idealismo por G.E. Moore. Stace no argumentó que el realismo sea falso sino solamente que no hay absolutamente ninguna razón para considerar que sea verdadero y por tanto no tenemos por qué creerlo. Para cualquier científico, su artículo "Science and the Physical World" (1935) es todo un reto intelectual. Puede parecer irónico que en pleno siglo XX un filósofo argumente que los átomos no existen, o que en todo caso nadie realmente podría saber si existen. Paradójicamente para muchos científicos, ésta es una discusión no concluida y de enorme actualidad filosófica -dos ejemplos del vigor y vitalidad de esta polémica son los siguientes: Roush, S. (2005) y Stanford P.K., (2006) - que esencialmente se centra en el análisis del realismo y de diferentes posturas anti-realistas.

\section{La ciencia y el mundo físico}

Stace inicia señalando que los científicos hablan de electrones, protones, neutrones y otras cosas que nunca percibimos directamente; por tanto, al preguntar ¿cómo sabemos que existen?, la única respuesta posible es que lo sabemos a partir de un proceso de inferencia de cosas que percibimos directamente. Pero -se pregunta- ¿qué clase de inferencia? Su respuesta es que es una inferencia causal en la que de algún modo los entes atómicos afectan a los organismos de modo tal que ellos pueden percibir el mundo cotidiano de mesas, sillas, etcétera. La única razón que tenemos para creer en la ley de la causalidad es que hemos observado ciertas regularidades o secuencias. Observamos que bajo ciertas condiciones A siempre es seguido de B y llamamos a A la causa y a B el efecto; por tanto la secuencia de causalidad A-B. Podemos notar que todas las secuencias causales observadas se dan entre objetos sensibles en el mundo de la percepción. La ley de la causalidad se aplica 
únicamente al mundo de los sentidos y no a nada más allá o atrás de él. Esto a su vez significa que no tenemos, ni jamás podremos tener, la mínima evidencia para creer que la ley de la causalidad pueda aplicarse fuera del mundo de la percepción. El hecho de que los átomos no sean inferidos a partir de los sentidos, implica necesariamente que de la existencia de sensaciones no podemos válidamente inferir la existencia de átomos. Esto implica que no podemos tener ninguna razón para creer en su existencia. Ésta es la razón por la cual Stace se propone argumentar que ellos no existen, o que en todo caso no podríamos saber si existen. Se sugiere no que la idea de átomo sea falsa o carente de sentido sino que éstos sólo son una abreviación ingeniosamente desarrollada por la mente humana que le permite hacer predicciones. Es importante subrayar que "predecir" aquí no se refiere exclusivamente al futuro pues igualmente podría predecirse que en el año 585 a. C. hubo un eclipse en Asia Menor.

Stace complementa su argumentación discutiendo el caso de la gravitación al señalar que Newton formuló su ley de gravitación en términos de "fuerzas". Se suponía que esta ley -que no es nada sino una fórmula matemática- gobierna la acción de estas fuerzas. Actualmente no se cree que estas fuerzas existan a pesar de lo cual la ley puede aplicarse para la predicción de fenómenos astronómicos; para el hombre de ciencia no es importante si esas fuerzas existen o no, eso, puede argumentarse es una pregunta filosófica pura. De hecho, Stace piensa que los filósofos considerarían a esas fuerzas como ficciones pero esto no invalida la ley, no la hace falsa o inútil, si puede ser usada para predecir fenómenos sigue siendo tan verdadera como siempre. Ahora sabemos que hay fallas en la ley de Newton y que otra ley debida a Einstein la ha sustituido. Se ha supuesto que la razón de esto es que ya no se cree en las fuerzas, sólo que éste no es el caso, dado que si las fuerzas existen o no simplemente no importa, lo que importa es que la ley de Newton 
no permite hacer predicciones exactas sobre algunos hechos astronómicos como, por ejemplo, la posición exacta del planeta Mercurio. La otra fórmula, la de Einstein, permite hacer las predicciones correctas. Esta nueva ley es una fórmula en términos geométricos, es matemática pura y nada más, no habla en ningún momento de fuerzas. Finalmente tampoco importa si las "lomas y valles en el espacio-tiempo de la teoría general de la relatividad” existen. La ley de Einstein es más verdadera que la de Newton no porque sustituye fuerzas por lomas y valles sino debido a que es una fórmula de predicción más precisa.

No solamente se puede decir que las fuerzas no existen sino que la "gravitación" tampoco. La gravitación no es una "cosa” sino una fórmula matemática que existe sólo en las cabezas de los matemáticos. Siendo una fórmula matemática no puede causar que un cuerpo caiga y en este mismo sentido la gravitación no hace que caiga un cuerpo. El lenguaje ordinario nos puede engañar, se habla de la ley "de" la gravitación y se supone que esta ley se "aplica a" los cuerpos materiales, debido a esta confusión equivocadamente suponemos que hay dos cosas, la gravitación y los cuerpos materiales, y que una de estas cosas, la gravitación, produce cambios en la otra.

En realidad nada existe excepto los cuerpos materiales, y en este sentido ni la ley de Newton ni la de Einstein son, estrictamente hablando, una ley de gravitación. Ambas son leyes que describen el movimiento de cuerpos, que nos dicen cómo esos cuerpos se mueven. Stace no deja de preguntarse por qué los seres humanos inventamos monstruos metafísicos como las fuerzas y las lomas y valles en el espacio-tiempo, su respuesta es que esto es debido a que los humanos no nos hemos emancipado de la absurda idea de que la ciencia puede "explicar" las cosas. No era suficiente decir "cómo" se mueven los planetas sino también se pretendía saber "por qué" se mueven del modo en que lo hacen. La respuesta de Newton fue, 
debido a "fuerzas". Las lomas y valles de la teoría de la relatividad general tienen la misma razón. Sin embargo las leyes científicas apropiadamente formuladas, nunca "explican" nada, simplemente establecen de modo abreviado y general qué ocurre. Ningún científico o filósofo sabe por qué las cosas ocurren ni puede "explicar" nada. Las leyes de la ciencia no hacen nada más que establecer el hecho bruto de que "cuando A ocurre, B siempre ocurre también". Las leyes de esta forma son las que nos permiten predecir. Si los científicos sustituyen fuerzas por colinas y lomas, únicamente están sustituyendo una superstición por otra.

Stace considera a los átomos de modo análogo a las fuerzas y a las lomas y valles del espacio-tiempo. Afirma que en realidad la formulación matemática, que es el modo científico de establecer la teoría atómica, está constituida por simples fórmulas para calcular lo que ocurre; sin embargo, así como la mente humana requiere que a la fórmula de gravitación le corresponda una "cosa" real que llamamos "gravitación" o "fuerza", así la misma debilidad humana exige que deba haber una cosa real que corresponda a la formulación atómica, a esta cosa le llamamos átomo. Esto aunque en realidad los átomos no causen sensaciones, ni la gravitación cause que las manzanas caigan. Lo único que puede causar una sensación es otra sensación y la relación de átomos a sensaciones no es una relación de causa a efecto sino la relación de una fórmula matemática a los hechos que le permiten al matemático realizar cálculos. La conclusión de la argumentación de Stace es que estrictamente hablando, nada existe excepto sensaciones (y las mentes que las perciben). El resto es solo una construcción mental y ficción. Esto no implica que concebir una estrella o un electrón sea falso o carezca de valor sino que su verdad y valor radica en su capacidad para ayudarnos a organizar nuestras experiencias y a predecir nuestras sensaciones. 


\section{Realismo e instrumentalismo}

Por una parte, hay construcciones humanas que son las teorías científicas y, por otra, tenemos el mundo al que esas teorías pretenden aplicarse (Brock, 2014; Psillos, 1999). ¿Cuál es la relación entre ambas? El enfoque, llamado realismo, afirma que el mundo es realmente como las teorías afirman. Por tanto, las partículas de la teoría cinética no son ficciones teóricas útiles sino átomos y moléculas con existencia real, y los campos eléctricos y magnéticos del electromagnetismo de Maxwell también son reales, así como las partículas con carga eléctrica que obedecen la ecuación de Lorentz. Un enfoque alternativo, llamado instrumentalismo, sostiene que los elementos teóricos de la ciencia no describen la realidad. Desde este punto de vista las teorías son sólo instrumentos de predicción y las partículas de la teoría cinética, los campos eléctricos y magnéticos de la teoría de Maxwell, así como las partículas con carga eléctrica que obedecen la ecuación de Lorentz, son nada sino meras ficciones.

Por lo general, el realismo se asocia con la idea de verdad pues para el realista la ciencia pretende dar descripciones verdaderas de lo que realmente es el mundo. $\mathrm{Al}$ instrumentalismo también se le asocia una idea de verdad pero más restringida ya que para éste una descripción del mundo observable es verdadera sólo si la descripción dada es correcta o no. Para el instrumentalista no es relevante si existen o no los elementos teóricos propuestos por una teoría sino solamente si la teoría describe o no el mundo observable. Para él la ciencia no es un medio para cubrir la distancia entre lo observable y lo inobservable.

La discusión filosófica del realismo y de diferentes formas de antirrealismo es una de las más activas y fructíferas de la filosofía de la ciencia. Preguntas como: ¿Deben las teorías científicas ser entendidas como descripciones verdaderas del mundo? ¿Debemos 
aceptar que las entidades que postulan las teorías realmente existen? Son fundamentales en este debate.

Por otra parte, los realistas conceden que muchas teorías aceptadas en el pasado han terminado siendo consideradas falsas, sin embargo insisten que de modo claro hay un progreso en la ciencia. Es bien conocido que entre realistas e instrumentalistas hay una cantidad de posiciones intermedias, tres ejemplos representativos son los siguientes: The Ontological Status of Theoretical Entities, de Grover Maxwell (1962) en donde se argumenta que la línea entre lo que es observable y no observable es en el mejor de los casos difusa; The Scientific Image, de Bas van Fraassen (1980), que argumenta contra la posición de Maxwell y formula su propio empirismo constructivo, de acuerdo a esta versión de antirrealismo uno puede aceptar teorías científicas y permanecer agnóstico sobre su verdad requiriendo solamente que las teorías sean empíricamente adecuadas o verdaderas acerca de los observables; por último, Alan Musgrave (1985) en su trabajo, Realism versus Constructive Empiricism, critica el enfoque de Van Fraassen señalando que uno de los más populares argumentos del realismo científico, el del "milagro" sobrevive la crítica de Van Fraassen. Brevemente, este argumento sostiene que el realismo es la mejor explicación para el éxito de la ciencia, si el realismo fuera falso nuestras teorías científicas serian un milagro.

\section{La evidencia científica}

Por lo menos, desde la época de los antiguos griegos se ha postulado la existencia de los átomos, esas esferas de materia duras e invisibles tan pequeñas que constituían los diferentes elementos de los que se forman los objetos materiales en el mundo físico. Esta idea fue despreciada durante muchos siglos hasta que volvió a surgir en diferentes formas y contextos durante la Revolución Cien- 
tífica del siglo XVII gracias a pensadores como Descartes, Boyle, Newton y Gassendi. Así mismo, la investigación del calor y de la química durante el siglo XIX aportó grandes apoyos a la existencia de estos supuestos átomos. Finalmente, a lo largo del siglo XX se obtuvo evidencia que muchos consideran como la confirmación de la existencia del átomo más allá de toda duda razonable.

A la pregunta: ¿Cómo sabemos que los átomos existen? Los científicos tienen, o afirman tener, muchas respuestas con evidencia sólida, algunas de las más importantes, en orden cronológico, son: 1) La ley de las proporciones definidas o Ley de Proust, 2) la teoría cinética de los gases, 3) el movimiento Browniano y 4) imágenes de microscopio de efecto túnel.

La ley de las proporciones definidas o ley de Proust fue obtenida a partir de las investigaciones químicas realizadas a finales del siglo XVIII y principios del XIX las cuales mostraban que los elementos participan en los compuestos en proporciones que siempre son expresables como números naturales asignados a cada elemento. Estos compuestos nunca presentaban la participación de un elemento como una fracción de una unidad, sugiriendo que son unidades indivisibles de cada elemento, que podemos llamar átomos. Por ejemplo, 1 unidad de sodio se combinaba con una unidad de cloro para obtener 1 unidad de sal (cloruro de sodio), pero nunca 1,5 unidades de sodio con 1,1 unidades de cloro. Esta ley, puramente experimental, recibió apoyo teórico cuando John Dalton publicó su teoría atómica en el tratado $A$ New System of Chemical Philosophy en 1808; también, cuando Amadeo Avogadro en 1811 propuso la hipótesis según la cual: "En volúmenes iguales de todos los gases, medidos en las mismas condiciones de presión y temperatura, existen igual número de moléculas", actualmente esto se enuncia como: "Un mol de diferentes sustancias contiene el mismo número de moléculas". El número de Avogadro se interpreta como el número de moléculas presentes en un mol de cualquier 
sustancia y se considera como fuerte evidencia de la existencia de átomos y moléculas.

La teoría cinética de los gases fue desarrollada a lo largo del siglo XIX. Las leyes de la termodinámica muestran el comportamiento macroscópico de las sustancias. De hecho, esta teoría no requiere considerar ninguna estructura microscópica para la materia. Sin embargo Clausius y Maxwell demostraron con la teoría cinética que la teoría atómica de la materia era capaz de dar cuenta de muchas propiedades de la materia que no pueden ser fácilmente explicadas de cualquier otra manera. La primera descripción sistemática de esta teoría fue dada por Rudolf Clausius en 1857 en su artículo titulado $O n$ the nature of the motion, which we call heat, en donde exitosamente deriva la ecuación de estado de un gas monoatómico. La ecuación de estado de la termodinámica de un gas ideal es una ecuación que relaciona parámetros como temperatura, presión y volumen y fue obtenida a partir de la suposición de que existen pequeñas partículas que pueden ser átomos o moléculas. Es decir; se pudo mostrar que las propiedades de los gases y el comportamiento del calor como una forma de energía en diferentes situaciones se podían explicar por los movimientos e interacciones de átomos que obedecen las leyes del movimiento de Newton. Así mismo, con la teoría cinética se obtuvo el calor especifico (la inercia térmica de un sistema) de gases formados por partículas de una sola especie, y los llamados coeficientes de transporte como la viscosidad, la conductividad térmica y el coeficiente de difusión. Partiendo de esta teoría, en 1860 James Clerk Maxwell publicó una serie de artículos titulados Illustrations of the dynamical theory of gases en donde da a conocer su ley de distribución de las velocidades moleculares, que es la primera ley estadística de la historia, la cual proporciona el número de moléculas cuya velocidad está en un rango dado. En 1877, Ludwig Boltzmann propone la segunda 
ley de la termodinámica entendida como una expresión del comportamiento estadístico de un gran número de partículas.

El movimiento browniano, comienza en 1905, cuando Albert Einstein demostró que la teoría atómica era capaz de dar una explicación detallada del movimiento puramente aleatorio de unos granos de polen microscópicos suspendidos en un líquido estacionario. El fenómeno, conocido como movimiento browniano cuando se trata de polen (su nombre general es pedesis), podía ser explicado asumiendo solamente que los granos suspendidos estaban sujetos a colisiones aleatorias por las más pequeñas moléculas del fluido, que impactan en el grano desde diferentes direcciones. Comparaciones entre las observaciones experimentales de los movimientos aleatorios realizadas por Perrin (1909) y las predicciones de Einstein demostraron una completa coincidencia. Esto ha sido considerado como una sólida prueba de la existencia de los átomos. Sin embargo ha sido señalado por Stanford (2009), que esto supone que la observación del movimiento Browniano es prueba suficiente de confirmación de la hipótesis atómica, esto último basado en la suposición de que "no hay ninguna otra hipótesis que pueda explicar el desplazamiento aleatorio de las partículas en el movimiento Browniano".

Imágenes del microscopio de efecto túnel (Scanning tunneling microscope o STM): El desarrollo tecnológico del siglo XX permitió en 1981 construir este instrumento el cual es capaz de tomar imágenes de superficies a nivel atómico. Sus inventores Gerd Binnig y Heinrich Rohrer (de IBM Zürich), recibieron por este trabajo el Premio Nobel de Física en 1986. El principio de operación está basado en el "efecto túnel" y consiste en colocar una aguja muy aguda cerca de la superficie a examinar. Cuando la punta conductora es colocada muy cerca de la superficie a ser examinada, una corriente de polarización causada por una diferencia de voltaje, aplicada entre las dos puede permitir a los electrones pasar al otro lado 
mediante efecto túnel a través del espacio entre ellas. La resultante corriente de tunelización es una función de la posición de la punta, el voltaje aplicado y la densidad local de estados electrónicos del átomo (LDOS por sus siglas en inglés) de la muestra. La información es adquirida monitoreando la corriente conforme la posición de la punta escanea a través de la superficie, y es desplegada en forma de imagen. Para un STM, una buena resolución es $0.1 \mathrm{~nm}$ de resolución lateral y $0.01 \mathrm{~nm}$ de resolución de profundidad. Con esta resolución, los átomos individuales dentro de los materiales son rutinariamente visualizados. En 1990, científicos del Centro de Investigación de Almaden en California, Estados Unidos, descubrieron como manipular la posición individual de átomos sobre superficies metálicas utilizando un microscopio de efecto túnel. La figura (tomada del sitio: http://www-03.ibm.com/ibm/ history/ exhibits/ vintage/vintage_ 4506VV1003.html) muestra las letras "IBM" construidas utilizando átomos individuales.

\section{Conclusiones}

Como se mencionó anteriormente, para el instrumentalista los modelos de la ciencia son sólo herramientas de predicción útiles que no nos dicen nada sobre lo que existe. No se dice que la idea de átomo sea falsa o carente de sentido sino que los átomos sólo son una ficción ingeniosamente desarrollada por la mente humana a través de modelos científicos que le permite hacer predicciones. Podemos ahora preguntar ¿cómo alguien que afirma lo anterior puede explicar la abrumadora evidencia que presenta el científico para mostrar la existencia de los átomos? Una forma de responder a esta pregunta se basa en el hecho (que contradice a la opinión inductivista ingenua) de que las teorías preceden a la observación. Esto debido a que los enunciados observacionales se hacen siempre en el lenguaje de alguna teoría y serán tan precisos como lo sea el 
marco conceptual o teórico que utilicen. Chalmers (1999), presenta el siguiente ejemplo, consideremos el enunciado observacional: "He aquí un trozo de gis" emitido por un profesor al tiempo que señala una barra cilíndrica blanca que mantiene delante del pizarón. Incluso este enunciado observacional tan básico conlleva una teoría y es falible. Se da por supuesta una generalización de muy bajo nivel tal como "las barras blancas que se encuentran en los salones de clase con trozos de gis". Y, desde luego, no es necesario que esta afirmación sea verdadera. El profesor de nuestro ejemplo puede estar equivocado. Puede ser que el cilindro blanco en cuestión no sea un trozo de gis sino una imitación cuidadosamente hecha, colocada allí por un alumno travieso que busca divertirse. El profesor o cualquiera de los presentes, podría dar un paso más para comprobar la verdad del enunciado "he aquí un trozo de gis", pero es muy significativo que cuanto más rigurosa sea la prueba, más se requiera de la teoría sin que esto implique alcanzar una certeza absoluta. Por ejemplo, al ser desafiado el profesor podría pasar el cilindro blanco a lo largo del pizarrón y señalar la línea blanca resultante afirmando: "Aquí lo ven, esto es un pedazo de gis". Sin embargo esto implica el supuesto de que "el gis deja una línea blanca cuando se hace un trazo en un pizarrón". Se podría contestar a la demostración del profesor diciendo que hay otras cosas, aparte del gis, que deja trazos blancos en los pizarrones. Ante esto el profesor podría pulverizar el gis y recurrir al análisis químico. Químicamente, el gis es en su mayor parte carbonato de calcio, afirmará el profesor, y por tanto producirá dióxido de carbono si se le sumerge en un ácido. Efectuará la prueba y mostrará que el gas resultante es dióxido de carbono mostrando que vuelve lechosa el agua de cal. Podemos ver que cada una de las etapas de esta serie de intentos por mostrar la validez del enunciado observacional "he aquí un pedazo de gis" conlleva a una apelación no sólo de nuevos enunciados observacionales, sino también a más generalizaciones 
teóricas. De hecho la prueba final dada supone un amplio conocimiento de teoría química como el efecto de los ácidos sobre los carbonatos y el efecto del dióxido de carbono sobre el agua de cal. Para establecer la validez de un enunciado observacional, por tanto, es necesario apelar a la teoría y cuanto más firmemente se haya de establecer la validez de la observación, mayor será el conocimiento teórico que se requiera. Aquí termina el ejemplo dado por Chalmers.

Vemos que demostrar la validez de la afirmación "he aquí un pedazo de gis" es bastante complejo, esto a pesar de que un gis es un objeto macroscópico que es percibido por nuestros sentidos. Demostrar la validez de la afirmación "los átomos existen" es doblemente complejo debido a que estamos hablando de entes que no son percibidos por nuestros sentidos y que son elementos teóricos que forman parte de complejas teorías científicas obtenidas a partir de largos y complicados procesos de razonamientos teóricos y experimentales. La ley de las proporciones definidas, la teoría cinética de los gases, el movimiento Browniano y las imágenes de microscopio de efecto túnel, son el resultado de la interpretación de observaciones experimentales dentro de ciertas teorías y de una cadena de inferencias, además de que no puede excluirse la posibilidad de que otras teorías con diferentes fundamentos puedan explicar las mismas observaciones experimentales, por remoto que esto parezca. Por ejemplo, una consecuencia de la hipótesis de Avogadro es que una unidad con el mismo peso atómico o molecular de toda sustancia contiene el mismo número de átomos o moléculas. Sin embargo la hipótesis de Avogadro fue ignorada por los químicos durante casi medio siglo hasta que en 1858 Stanislao Cannizaro convenció a la mayoría de la comunidad científica sobre la simplicidad para interpretar las observaciones a partir de la hipótesis atómica. La termodinámica no requiere suponer ninguna 
estructura para la materia y fue sorprendente derivar la ecuación de estado de la termodinámica a partir de la teoría cinética.

No obstante, aún había algunos que sugerían que si bien la hipótesis atómica era útil y conveniente eso no la hacía necesariamente válida. Efectivamente, todas las pruebas mencionadas requieren de la aceptación de una gran cantidad de hipótesis que deben de ser aceptadas para posteriormente concluir que la más plausible explicación es precisamente la existencia de átomos. En el artículo, Can Theories of Chemistry Provide an Argument Against Realism? (Gavroglu, 1996) se señala que Neville Sidgwick, al publicar su libro The Electronic Theory of Valency-escrito en 1926 justamente después de los importantes desarrollos teóricos de Schrödinger y Heisenberg-, claramente mostraba su entusiasmo por la nueva mecánica cuántica pero sin comprometerse con la existencia de las entidades propuestas. Se menciona que:

Al desarrollar una teoría de Valencia los químicos tienen dos alternativas: Usar símbolos sin una connotación física definida para expresar la reactividad de los átomos en una molécula y dejar que el progreso futuro de la ciencia descubra que realidad representan esos símbolos, o adoptar los conceptos de la física atómica -electrones, núcleos, orbitas- para tratar de explicar la realidad química.

No se puede negar que aceptar la existencia de átomos simplifica enormemente la interpretación y manejo de modelos teóricos, así como de observaciones experimentales; sin embargo, siempre sería posible diseñar una explicación y presentar modelos alternativos con los cuales se podría evitar recurrir a la hipótesis atómica. Es seguro que la mayoría de los científicos considerarían este ejercicio como una actividad ociosa y redundante, no obstante, el punto fundamental desde un enfoque filosófico es el hecho de que, a pesar de la abundante evidencia disponible, no hemos visto átomos 
sino sólo resultados experimentales que concuerdan -de manera adecuada, se debe aceptar- con modelos teóricos y experimentales basados en la hipótesis atómica. Suponer que no hay alternativas explicativas posibles para un conjunto de observaciones más allá de las actualmente conocidas es un error lógico argumentativo además de histórico, ejemplos importantes de esto son los siguientes: 1) James Clerk Maxwell afirmó (1873) que no había alternativa a postular la existencia del éter para explicar la propagación de ondas. En su Tratado de Electricidad y Magnetismo, Maxwell escribe: "Siempre que la energía es transmitida de un cuerpo a otro en el tiempo, debe de haber un medio o sustancia del cual la energía sale de un cuerpo antes de entrar al otro". Ahora sabemos que el éter propuesto por Maxwell no existe y esto sólo confirma que había posibilidades teóricas no concebidas y desconocidas por él y por todos los científicos de su época. 2) Lavoisier argumentó en su defensa de su Teoría del Calor (1785) que el fluido llamado calórico debe de existir para poder explicar cómo son producidos una serie de fenómenos termodinámicos. En su "Memoir on Phlogiston" afirma: "Difícilmente podemos pensar en esos fenómenos sin admitir la existencia de un fluido especial (cuya acumulación causa calor y cuya ausencia causa frio)". Posteriormente en su Traité de Chimie publicado en 1789, escribe: "Es difícil concebir estos fenómenos (observaciones) sin admitir que ellos son el resultado de una sustancia real y material, un fluido sutil". Nuevamente, ahora sabemos que el calórico propuesto por Lavoisier no existe, lo cual una vez más confirma que había posibilidades teóricas desconocidas, no concebidas por él y los científicos de su época. Por remoto que parezca, existe la posibilidad lógica de que no concebidas propuestas teóricas expliquen los resultados observacionales actuales sin requerir de la hipótesis atómica. 


\section{Referencias}

Brock, S., 2014, Realism and Anti-Realism (Central Problems of Philosophy), Routledge, Nueva York.

Chalmers, A.F., 1999, What is that thing called science?, Hackett Publishing, Londres.

Clausius, R., 1857, "Über die Art der Bewegung, die wir Wärme nennen (On the nature of the motion, which we call heat)", Annalen der Physik, núm. 176, pp. 353-380

Dalton, J., 1808, A New System of Chemical Philosophy, R. Bickerstaff, Manchester.

Einstein, A., 1905, "Über die von der molekularkinetischen Theorie der Wärme geforderte Bewegung von in ruhenden Flüssigkeiten suspendierten Teilchen (On the motion of small particles suspended in stationary liquids required by the molecular-kinetic theory of heat)", Annalen der Physik, vol. 4, núm. 17, pp. 549-560.

Gavroglu, K., 1996, "Can Theories of Chemistry Provide an Argument Against Realism?”, En R. S. Cohen, R. Hilpinen y QiuRenzong (eds.), Realism and Anti-Realism in the Philosophy of Science, Kluwer Academic Publishers, pp. 149-170.

Lavoisier A., 1785, Ouvres de Lavoisier, vol. 1-4, Imprimerie Impériale, París.

Mawell, J.C., 1873, A treatise on Electricity and Magnetism, Clarendon Press, Oxford.

1960, "Illustrations of the dynamical theory of gases", Philosophical Magazine, núm. 19, pp. 19-32.

Maxwell, G., 1962, The Ontological Status of Theoretical Entities, Scientific Explanation, Space, and Time, vol. 3, University of Minnesota Press, Minnesota Studies in the Philosophy of Science, Minneapolis. 
Musgrave A., 1985, "Realism versus Constructive Empiricism”, en Images of Science, Chicago University Press, Chicago.

Perrin J., 1909, "Le Mouvement Brownien et la Réalité Moleculaire”, Annales de Chimie et de Physique, núm. 18, pp. 5-114.

Psillos, S., 1999, Scientific Realism: How Science Tracks Truth (Philosophical Issues in Science), Routledge, Londres.

Roush, S., 2005, Tracking Truth, Oxford University Press, Oxford.

Stace, W.T., 1920, A Critical History of Greek Philosophy, First Rate Publishers, Londres.

, 1924, The Philosophy of Hegel: A Systematic Exposition, Kessinger Legacy Reprints, Montana.

, 1929, The Meaning of Beauty: A Theory of Aesthetics, Grant Richards and Humphrey Toulmin, Londres.

, 1932, The Theory of Knowledge and Existence, The Clarendon Press, Oxford.

, 1935, Science and the Physical World.

dres.

, 1960, Mysticism and Philosophy, Palgrave Macmillan, Lon-

Rudge D., 1998, Philosophy of Science, Prometheus Books, Londres.

Stanford, P.K., 2006, Exceeding Our Grasp, Oxford University Press, Nueva York.

, 2009, "Scientific Realism, the Atomic Theory, and the Catch-All Hypothesis: Can We Test Fundamental Theories Against All Serious Alternatives?", British Journal for the Philosophy of Science, núm. 60, pp. 253-269.

Van Fraassen, Bas C., 1980, The Scientific Image, Clarendon Press, Oxford. 


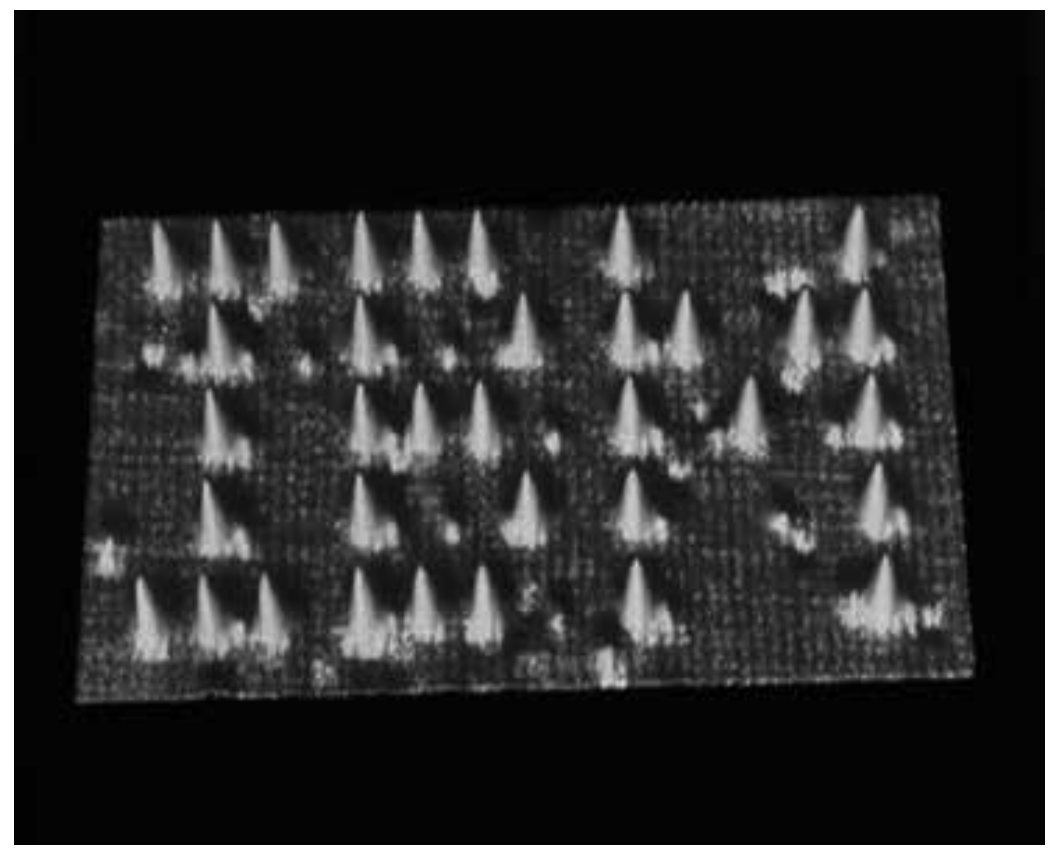

Figura

Imagen de átomos obtenida con un microscopio de efecto túnel. Disponible en: http://sysrun.haifa.il.ibm.com/ibm/history/exhibits/vintage/ vintage_4506VV1003.html

Recibido: 21 de marzo de 2017 Aceptado: 3 de mayo de 2017 